\title{
A Network Selection Method for Handover in Vehicle-to-Infrastructure Communications in Multi-Tier Networks
}

\author{
Emmanuel Ndashimye ${ }^{1}$, Nurul I. Sarkar ${ }^{2}$ and Sayan Kumar Ray ${ }^{3}$
}

\begin{abstract}
Network selection is very important for a successful handover in a multi-tier heterogeneous networks. However, the primary challenges currently faced by research community is the lack of availability of network information at the mobile node side for efficiently select the most appropriate target network. It is practically difficult for an UE to get network information from base stations/access point of the neighbouring networks before connecting to them. In response to this, this paper proposes a network selection method that applies the knowledge of mobility data and the network load information to carry out an efficient handover for vehicle-to-infrastructure (V2I) communication over multi-tier heterogeneous networks. We first derive key parameters, such as relative direction index, proximity index, residence time index, and network load index to select the best candidate network. A moving vehicle would be able to select the most appropriate target network by selecting one or more of the above parameters. We then test our algorithms by developing a dual mode vehicle OnBoard Unit (OBU) equipped with both Long Term EvolutionAdvanced (LTE-A) and $\mathrm{Wi}-\mathrm{Fi}$ network interface cards in OPNET simulator. The performance of the proposed handover method is evaluated by extensive OPNET-based simulation experiments. In the simulation model, we consider a multi-tier heterogeneous network comprising of a macro and multiple small cells of LTE-A and IEEE 802.11n technologies. Results show that our proposed handover method offers about $50 \%$ higher throughput and up to $43 \%$ higher packet delivery ratio than the conventional received signal strengths (RSS) based network selection method.
\end{abstract}

Keywords: V2I communication, Multi-tier network, LTE-A, Wi-Fi, Handover.

\section{INTRODUCTION}

The Fifth Generation (5G) wireless networks support seamless coexistence of different types of radio access technologies (e.g. LTE, Wi-Fi, WiMAX, IoT Low Power Wide Area Networks) in multi-tier network scenarios [1]. There will be network densification with the macro-cells overlaying the plethora of heterogeneous small cells of different coverage areas (e.g., femto and pico cells). The advantages of deploying small cells in 5G networks are multifold, which may include provision of good quality of service, increase in traffic capacity and data rate, more capacity through macro cell traffic offloading and lower fees. Moreover, small cells can provide network services to areas with patchy or no macro cell signal coverage [2]. However, the presence of multiple underlying networks with different coverage areas,

\footnotetext{
${ }^{1}$ N. Emmanuel and ${ }^{2}$ N.I.Sarkar are with Department of IT and Software Engineering, Auckland University of Technology, Auckland, New Zealand \{endashimeaut.ac.nz

${ }^{3}$ S.K.Ray is with Faculty of Business and Information Technology Manukau Institute of Technology, Auckland, New Zealand. Sayan.Ray@manukau.ac.nz
}

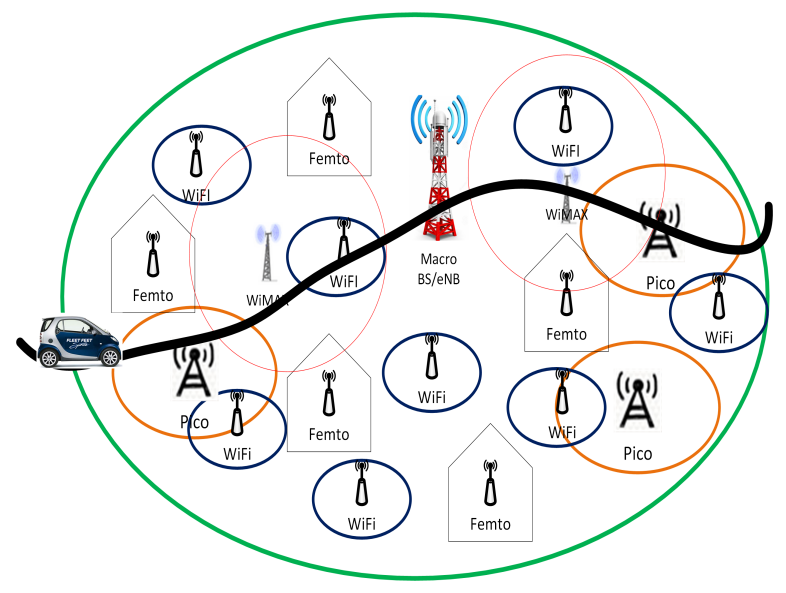

Fig. 1. A typical V2I communication over multi-tier heterogeneous wireless networks

characteristics and QoS requirements can pose challenges for high-speed vehicles [3].

For an effective vehicle-to-infrastructure (V2I) communication in an urban multi-tier network environment, vehicles on the move will need to perform fast and successful handovers not only between macro and small cell networks, but also within small cell networks to ensure seamless mobility and communication. The deployment of small cell networks is increasing and has raised a number of challenges in regards to performing handover from one network to another. These challenges include possible frequent unnecessary handovers, ping-pong effects, unsuccessful handovers and call drops, signalling overheads and resource wastages [3][4]. These issues can hamper the overall handover performance in V2I communications. Figure 1 illustrates a typical V2I communication scenario with a moving vehicle passing through a multi-tier heterogeneous urban network environment.

While maintaining fast handover for seamless mobility is important, selecting the most appropriate target network is also critical for a successful and efficient handover. An inappropriate selection of target network for handover may lead to packet losses and even a handover failure (HOF). In V2I communications, one of the primary reasons for unnecessary and unsuccessful handovers (including call drops) is the vehicles incorrect selection of the target network for handover in the node mobility. Selection of the most appropriate target network has always been a challenging task even for a conventional single-tier cellular network where handover is based on received signal strengths (RSSs) from multiple neighbouring target networks. This will be even 
more complicated in $5 \mathrm{G}$ networks where various networks of different sizes coexist. Selection of the most appropriate target network in such cases ideally should be based on multiple parameters. The handover methods and target network selection over single-tier heterogeneous networks have been widely studied by many network researchers [5][6]. However, the existing network selection methods are not suitable for high-speed vehicular handover in multitier heterogeneous networks. To select the most appropriate target networks for handover, we propose a network selection method to facilitate fast and successful handover for effective V2I communications in an urban multi-tier network environment.Our approach builds on the concept of Access Network Discovery and Selection Function (ANDSF) [5] which is introduced in Release 8 of the Third Generation Partnership Project (3GPP), located in Evolved Packet Core (EPC) of LTE systems. ANDSF selects the target network in heterogeneous networks where a vehicle OBU can discover and select the underlying access policies and parameters obtained from the ANDSF server [7].We derive key network parameters such as relative direction index, proximity index, residence time index, load index and the received signal strength. Based on network selection information from an ANDSF server, the vehicle initiates the network selection process for a potential handover well in advance before approaching the coverage area of the target cell.The following terminologies are used in this paper: an OBU implies an in-vehicle communication device that can connect and communicate to the roadside infrastructure networks; small cell access points (SAPs) represent both LTE-A small cell eNBs and Wi-Fi APs.

In our previous work [4], we presented the basic characteristics of V2I communications in heterogeneous multi-tier network environments. Also, we have provided a comprehensive survey on several V2I research challenges. In this paper, we address the issue of selecting an appropriate underlying network for an effective V2I communication over multi-tier heterogeneous networks. The main contributions and strength of this paper are:

- An analytical model is developed to derive relative direction index, proximity index and residence time index in order to short-list of candidate network for vehicle with a reasonable coverage.

- By considering network load at candidate networks, we investigate the capability of accommodating new handover sessions. For each short-listed candidate network, a network load index is analytically derived based on the number of users connected. The network load index is primarily used for minimizing the handover blocking probability.

- We develop and test our network selection algorithms in OPNET simulator. A dual-mode vehicle On-Board Unit (OBU) containing both Long Term EvolutionAdvanced (LTE-A) and WiFi network interface cards is also developed by writing $\mathrm{C}++$ code in OPNET simulator. This allows us to evaluate and validate the

\section{system model.}

The paper is organized as follows. Section II reviews literature on V2I communication and handover selection methods. The proposed network selection method is presented in Section III. The system simulation model and results are presented in Section IV, and a brief conclusion in Section V ends the paper.

\section{RELATED WORK}

Selection of the most appropriate underlying network for handover is critical for a moving vehicle to maintain an ongoing communication seamlessly. Such selections become more challenging in environments where networks of different sizes (multi-tier) and different technologies (heterogeneous) coexist. Many researchers have attempted to address the issues of selecting the most appropriate networks for handover in multi-tier heterogeneous environments. The recent standardization initiatives have also addressed the issues of handover. In this paper, for brevity, we refer to only a selected set of literature that is indicative of the range of approaches used to select the appropriate networks for handover and related issues.

Motivated by the high data rate of Wi-Fi networks, 3GPP has defined different methods of integrating cellular and WiFI technologies. In this regard, in its releases $8,10,11$ and 12 [7] [8], the Third Generation Partnership Project (3GPP) has also introduced ANDSF that primarily enable radio access discovery in heterogeneous networks. This has advanced the network selection process to the extent of supporting the UE to discover, select and connect to both non-3GPP and 3GPP access networks (UTRAN, LTE and HSPA). ANDSF facilitates network selection by providing the UE with the list of networks that may possibly be in its service area along with access polices [8]. ANDSF was applied by some researchers $[9,10]$ for selecting the best network for handover activity. In [9], ANDSF was used in combination with software-defined network (SDN) to suggest selection of most suitable target network in LTE - Wi-Fi heterogeneous network. In this proposal, ANDSF server provides information about overloaded eNBs so that are excluded from candidate list of target cells. Similarly, the approach proposed in [10], ANDSF has been utilized for efficient handover management such as network resource balancing between LTE and Wi-Fi [10].

Similar to initiatives from 3GPP, the Wi-Fi Alliance has introduced the Hotspot 2.0 as part of the IEEE 802.11u standardization initiatives to improve the experience of Wi-Fi roaming [11]. Wi-Fi devices can utilize information available from Hotspot 2.0 aims to assist Wi-Fi devices to automate the process of underlying Wi-Fi network discovery, selection, authentication, registration and connection to the most suitable and available WiFi hotspots in the region [12]. ANDSF and Hotspot 2.0 have complementary aspects that can fill up individual shortcomings, therefore their combination could provide a seamless integration of cellularWi-Fi technologies, and thus enhancing the user experience [13]. 
To prevent the unnecessary handovers, a number of studies have suggested that fast moving vehicles associate with macro-cell while low-speed ones are served by small cells [14]. Based on the speed of the movement and the estimated coverage area of the candidate networks, the author of [15] designed a network selection mechanism that instructs the high-speed vehicles to select macro-cell eNBs while lowspeed vehicles are taken care by the micro-cells. These methods can easily enable the mobile node to determine and select the candidate network for a handover, however, the high-speed mobile node may experience the increased handover dropping probability when the macro-cell base stations are overloaded. This issue was addressed in [16] by combining the knowledge of node speed and the QoS requirements. In this approach, UEs with high speeds are associated with macro-cell to reduce the number of handovers, while UEs with high requirements of QoS are associated with pico-cells for minimizing the packet loss ratio. However, the authors overlooked special UEs with high speed and high QoS requirements. Moreover, assigning UEs with high requirements of QoS could degrade the service quality due to multiple frequent handovers.

To account for mobility, mobile users are classified as fast and slow based on the dwelling period of the node within the coverage areas of the considered cells and thus they are assigned to macro-cell or small-cells accordingly [17]. The dwelling period is determined based on the cell dimensions and the mobility information such as direction and the user trajectory [18]. While selection approaches proposed in [17] and [18] could optimise network selection for an efficient handover, the shortage of network resources at target network could result in handover drop that leads to radio link failure (RLF). It is important to consider bandwidth requirements before initiating any handover request [19]. Authors in [19] applied fuzzy logic to developed network selection mechanism that combines the knowledge of network load and the vehicle movement trend for selecting the appropriate network in a multi-tier networks. Owing to the associated complex mathematical techniques, these methods, however, suffered from an increased processing delay affecting the overall handover latency.

With the proliferation of small cells, appropriate selection of the underlying network to provide the best handover performance in a heterogeneous multi-tier network environment is becoming increasingly challenging and remains an open research issue. Most of the proposed research methods in this context considered either non-heterogeneous networks in a multi-tier environment (e.g., networks consisting of only LTE-Advanced macro and micro cells) or are designed for low speed mobile users such as pedestrians. Furthermore, most of these proposed methods did not explain how information about the various parameters used to select the underlying network for handover are collected from the involved mobile devices and base stations and how the information flowed through the system. On the contrary, the network selection method proposed in this paper considers a future $5 \mathrm{G}$ heterogeneous multi-tier network environment consisting of LTE-Advanced and WiFi radio technologies. A vehicle having an OBU, moving at high speed, can self-select the most appropriate underlying network for a handover activity based on multiple decision parameters. The method explains how information related to these parameters can be obtained by the vehicle based on realistic communication scenarios and how the information can be utilised to decide on the underlying network to be selected. The proposed network method is described next.

\section{Proposed Network Selection Method}

This paper proposes a novel network selection method for effective V2I communications in a multi-tier heterogeneous urban network environment. The basic idea was published in [18]. In addition to handover parameters considered in [18], the handover technique in this paper applies the knowledge of the available network load for selecting the most appropriate target network for an anticipated handover. In the proposed method, a vehicle with a dual-mode OBU (having LTE-A and Wi-Fi interfaces) travelling across the multi-tier network of macro and small cells is able to self-select the most appropriate target network for a potential handover based on multiple parameters.

These parameters include relative direction index, proximity index, residence time index, load index and received signal strength. The main purpose of relative direction index is to eliminate the useless candidate networks (those lying in the opposite direction of the movement of the vehicle). These candidate networks are eliminated from the set of candidate to be evaluated. The proximity index estimates the closeness of the candidate network to the trajectory of the movement. This is done by calculating the angular displacement of the vehicle relative to the physical location of candidate networks. This proximity index is eventually used to calculate the residence time index which determines the duration that the vehicle spends in the service area of the selected network. Load index computes the available network capacity while the RSS indicates the quality of the received signal from the candidate network base station/access point.

By self-selecting the most appropriate target network, the vehicle is able to not only reduce the number of unnecessary and unsuccessful handovers, but also improve the resources consumed and overall performance of mobility. Moreover, by performing a fast handover, it also improves the overall handover latency. Let us elaborate these advantages here. Firstly, in the proposed method, the vehicle only scans those neighbouring networks (from here onwards to be termed as candidate networks) that lies on the movement direction trajectory of the vehicle and do not scans other candidate networks. This reduced scanning implies less handover time or faster handover. Secondly, because of the multiple parameters considered, the vehicle can successfully choose the most appropriate target network for handover and therefore reduce the number of unnecessary and unwanted handovers including ping-pong effects. The proposed method provides a better handover performance as a result of reduced signalling overheads. 


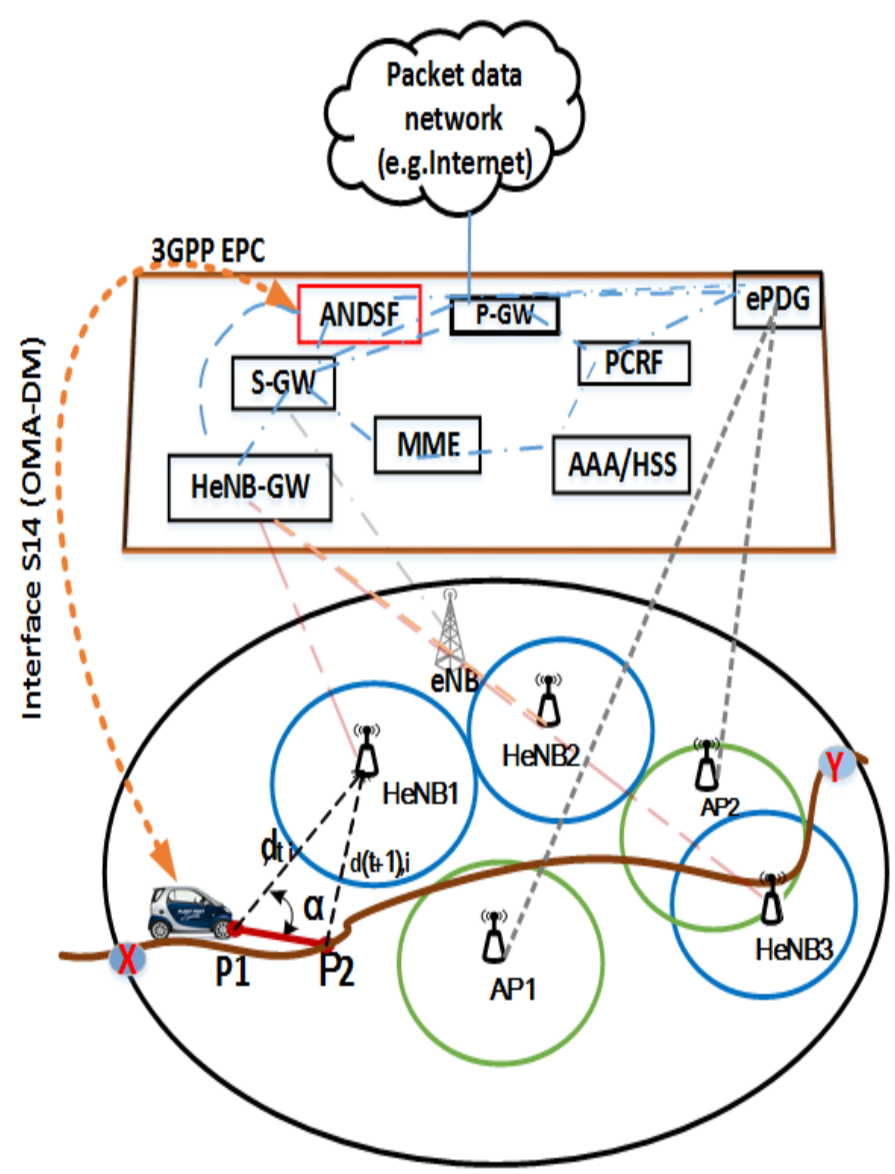

Fig. 2. The system model. LTE-A eNB overlays multiple LTE-A small cells and WiFi access points

Figure 2 shows the system model of the multi-tier heterogeneous network with one LTE-A macro cell having an Evolved Node B (eNB) and a mix of LTE-A small cells (femto/pico) and Wi-Fi access points (APs). The small cells (SC) are represented by HeNB and AP. The LTE-A macro network covers the entire service area and overlays the small cell networks, of limited coverage areas. In the overlapped areas between LTE-A SCs and Wi-Fi networks, vehicles with dual-mode (LTE-A and Wi-Fi) OBUs have multiple network options to choose from for handover. For example, referring to Fig. 2, vehicles under the service area of small cell $A P_{2}$ can handover either to the LTE-A macro-network or to another SC network provided that the target network (for handover) has adequate resources to support an ongoing communication.

\section{A. System Model}

In our proposed model, the ANDSF records the network context information (e.g., micro-cells SAPs location and access policies) and shares that with the vehicle OBU upon request [7]. The ANDSF server interacts with ANDSF clients, running inside individual OBU, through OMA-DM (Open Mobile Alliance Device Management) protocol over the S14 interface, which supports both push and pull data communi- cation mechanisms [17]. By referring to Fig. 2, the OBU on board the vehicle, moving from $\mathrm{X}$ to $\mathrm{Y}$, periodically queries the ANDSF about the geographical location and access policies of different networks available in its vicinity. While, access policies enable the OBU to identify the networks that provide open access based on inter-system mobility policy (ISMP) [14]. Geographical location information helps the vehicle to select those networks lying in its future trajectory and store them as potential candidates for future handover. In this work, however, we do not consider the access policyrelated information and assume that all small cell networks offer open access. We also assume that each OBU knows its instantaneous geographical location coordinates by means of Global Positioning Systems (GPS) [9].

The proposed network selection method operates in two stages. In the first stage, the vehicles OBU pro-actively makes a short list of potential target SAPs for handover based on three parameters, including relative direction index, proximity index and residence time index. In the next stage, the OBU selects the most promising candidate target among the shortlisted SAPs, based on load index and received signal strengths. The above handover parameters are described next.

\section{B. Relative direction index}

A preliminary idea of this parameter was given in [20] and this work employs a modified version of that. Relative direction index evaluates the movement direction of the vehicle relative to the geo-location of a candidate SAP based on distance calculation. Let us consider, two sequential positions, $\mathrm{P} 1$ and $\mathrm{P} 2$, of the vehicle along the movement trajectory (Refer to Fig. 3). At each of these positions, the vehicle OBU calculates its distance from all available $S A P_{i}$ (based on the Heversine formula [9]), which assumes the spherical form of the Earth and calculates the great-cycle distance between any two points on Earth. If $\left(\operatorname{Lat}_{v}, \operatorname{Lon}_{v}\right)$ and $\left(\operatorname{Lat}_{S A P i}, \operatorname{Lon}_{S A P i}\right)$, respectively, denote the coordinates of the vehicle and of the candidate $S A P_{i}$, and $\Delta_{l a t}$ and $\Delta_{l o n}$, respectively, denote the latitude and longitude separations, then the distance (d) between the vehicle and candidate $S A P_{i}$ can be calculated as:

$d=2 R \sin \sqrt{\sin ^{2}\left(\frac{\Delta_{l a t}}{2}\right)+\cos \left(l a t_{v}\right) * \cos \left(l a t_{S A P_{i}}\right) * \sin ^{2}\left(\frac{\Delta_{l o n}}{2}\right)}$

where the angles are in radians and $R=6371 K m$ is the radius of the Earth.

Next, we calculate $d_{i}$ the difference between two consecutive distances, $d_{(t, i)}$ measured at the current position $P_{2}$ and $d_{(t-1, i)}$ measured at previous position, $P_{1}$ of the vehicle as shown in Eq. 2.

$$
\Delta d_{i}=d_{t-1, i}-d_{t, i}
$$

This indicates the direction of movement of the vehicle relative to the each surrounding SAP. While $d_{i}>0$ implies a vehicle is moving towards the coverage area of $S A P_{i}, d_{i}<0$ indicates it is moving away from it and all SAPs showing regressive movement with respect to the vehicles direction of 
motion are omitted from the shortlisted potential candidate set, $N_{i}$. Thus, the probability of retaining $S A P_{i}$ in $N_{i}$, can be calculated as:

$$
f\left(\Delta d_{i}\right)= \begin{cases}1, & \Delta d_{i}<0 \\ 0, & \Delta d_{i} \geq 0\end{cases}
$$

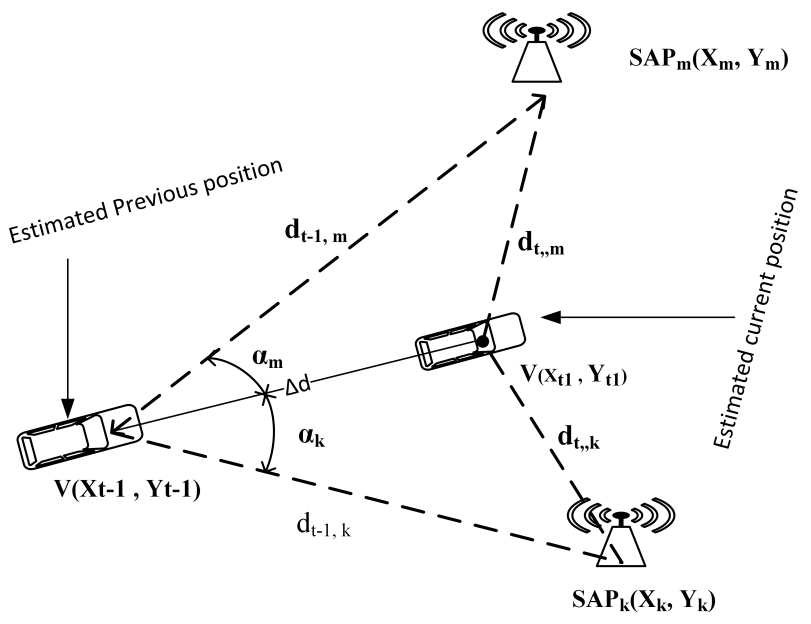

Fig. 3. Estimating the direction of relative movements

\section{Proximity index}

We introduce this parameter to calculate the proximity of a SAP to the movement trajectory of the vehicle. The proximity index $\omega_{i}$ indicates how near the target SAPs are to the movement trajectory of the vehicle and it is related to the angular displacement $\alpha_{i}$ of the vehicle (see Fig. 2). The factor $\cos \alpha_{i}$ denotes the proximity index calculated as per Equation (4).

$$
\omega_{i}=\cos \alpha_{i}=\frac{d_{t-1, i}^{2}+\Delta d^{2}-d_{t, i}^{2}}{2 * d_{t-1, i} * \Delta d}
$$

It is worth noting here that the angle $\alpha_{i}$ is restricted between 0 and $\pi$, since only those SAPs are considered with respect to which the vehicle shows progressive movement.

\section{Residence time index}

Residence time is another important parameter proposed in this work. It is the duration that the vehicle spends within the service area of a candidate SAP and is dependent on the vehicular speed and the coverage radius of the SAP. The residence time index $\left(t_{r}\right)$ is defined as the function of proximity index $\left(\omega_{i}\right)$, speed of vehicle $\left(V_{\text {Speed }}\right)$ and the maximum coverage radius $\left(C_{r}\right)$ of the candidate SAP.

$$
t_{r}=\frac{C_{r} * \cos \alpha_{i}}{V_{\text {speed }}}
$$

\section{E. Network load index}

This parameter indicates a SAPs capability to accommodate the new handover sessions. Having inadequate resources in the candidate network may result in a handover drop. To maintain load balancing, in this work, a candidate network is evaluated based on its load ratio (available capacity / total capacity) instead of its leftover bandwidth [6] as is commonly done. Equation (6) calculates the available bandwidth of the candidate $S A P_{i}$, while the load index $(l i)$ is represented in equation (7).

$$
\begin{gathered}
B_{r}=B_{A P i}-\sum_{u j \subset V_{A P_{i}}} b_{i j} \\
l_{i}=\frac{B_{r}}{B_{A p i}}
\end{gathered}
$$

In (6), $b_{i j}$ denotes the bandwidth required for user $u_{j}$, $V_{A P i}$ represents the set of all users currently connected to $S A P_{i}$, while $B_{r}$ and $B_{A P i}$ indicate the available and maximum bandwidth of the candidate $S A P_{i}$, respectively.

Referring to Fig. 2, $A P_{2}$ and $H e N B 3$ are the two potential target SAPs evaluated based on the above-mentioned parameters. Finally, the OBU in the vehicle measures the RSS of the potential candidate SAPs (in this case $A P_{2}$ and $H e N B 3)$ to make a final selection. Thus, the most appropriate candidate network $\left(n^{*}\right)$ for handover is selected as per (8). Algorithm 1 details then proposed network selection procedure.

Figure 4 presents the detailed messaging and procedure of the proposed network selection method. The figure shows one macrocell serving eNB (SeNB) overlaying multiple SAPs. The SAPs are able to report their geographical locations to the ANDSF server located in EPC network. As illustrated in the figure, the proposed network selection method consists of three steps. The first step involves the duplex communication established between the ANDSF client agent on the vehicle OBU (initially connected to SeNB), and the ANDSF server located in the EPC. Through this communication, the ANDSF server shares with the vehicle OBU information about the geographical locations of all neighboring candidates SAPs. The OBU then short-lists the potential candidate SAPs based on the relative direction index that it calculates on the basis of the received location information. It is worth mentioning here that only those SAPs that lie in the direction of movement of the vehicle make it to the shortlist. Next, the vehicle OBU computes the residence (dwelling) time for each of short-listed SAPs and based on that all shortlisted SAPs are ranked in descending order with the SAP having the maximum residence time getting the highest ranking. Finally, the vehicle OBU decides the most appropriate SAP based on parameters like the network load and received signal strengths (RSS).

To reduce both the chances of call drops and the number of unnecessary handovers, in the proposed method, the vehicle OBU performs the first two steps before the vehicle enters the coverage area of a potential target SAP. This is because once inside the coverage area of a SAP, depending on the speed of its movement, the vehicle will get very nominal time to perform all the three steps and complete the handover with the SAP before losing out on the connectivity. Moreover, as the vehicle OBU only initiates the handover activity with the most potential SAP decided on the basis of the proposed 


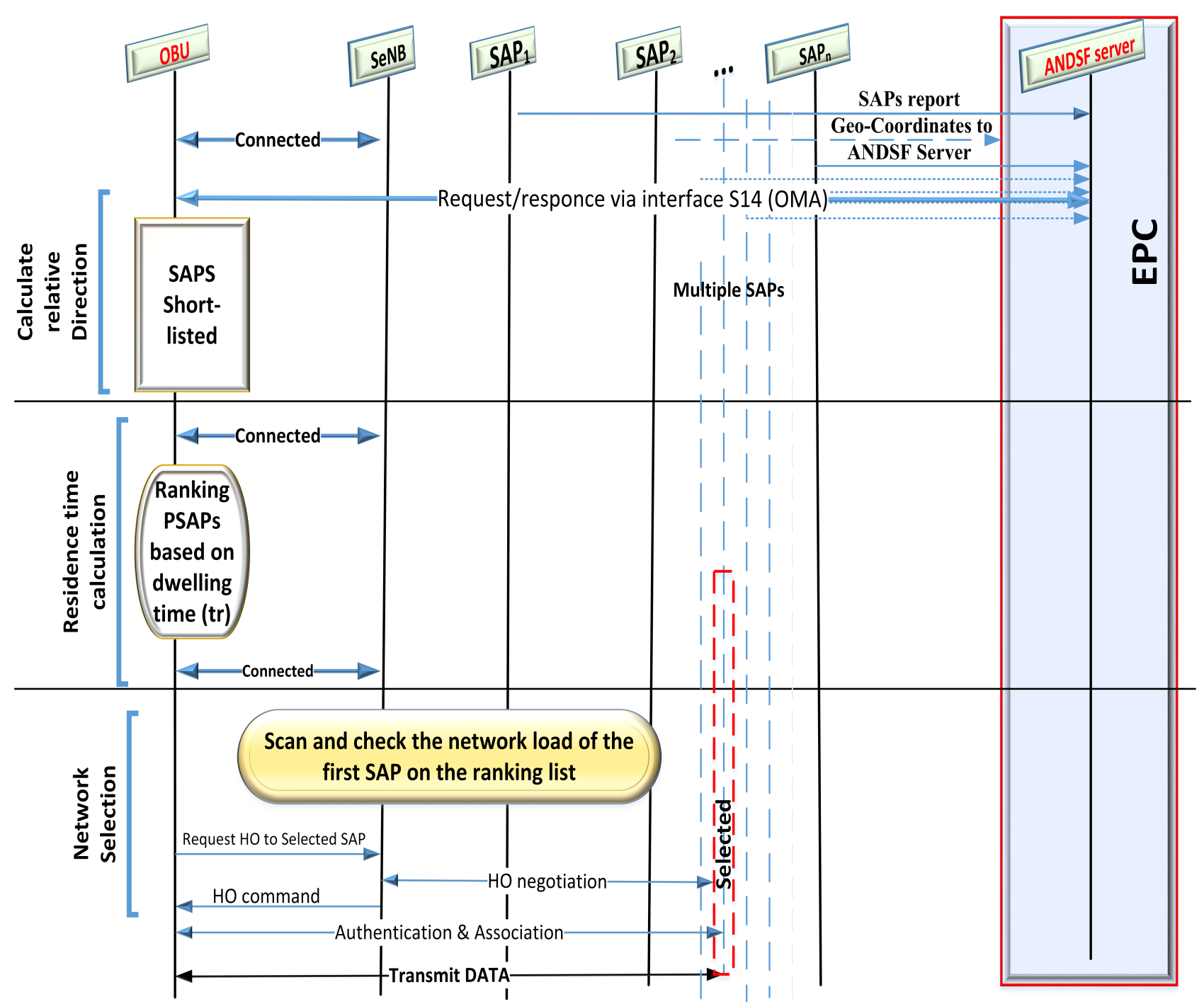

Fig. 4. Messaging and procedure of the proposed network selection method for handover

method, the overall signaling overhead of the system is improved.

\section{PERFORMANCE EVALUATION OF THE PROPOSED METHOD}

The proposed network selection method is evaluated through simulation studies performed in Riverbed (OPNET Modeller) network simulator (version 18.0) [21]. This section provides the details of the designing of the vehicle OBU node and then analyses the effectiveness of the proposed network selection method in terms of handover performance and QoS provision.

\section{A. OBU Node Design}

Figure 5 shows the multi-mode OBU that we have designed to evaluate the performance of the proposed method. The multi-mode OBU has two physical interfaces, LTE-A and Wi-Fi media access technologies (MAT). Figure 5(a) shows the OBU node's TCP/IP protocol stack prior to the inclusion of the proposed method. The modified OBU node is shown in Figure 5(b). The proposed network selection method implemented in the module VHO mgr (as shown in Figure 5(b)) is used to choose the appropriate RAT interface for the $\mathrm{OBU}$ to connect to based on Equation (8). The IP layer in the figure forwarded data only to the selected RAT interface. To ensure seamless connectivity, each of the two interfaces, LTE-A and Wi-Fi, involved in the vertical handover process is configured with a static route to the destination. In this context, it is worth noting that the proposed network selection method can be used not only for LTE and Wi-Fi MATs, but also for any other types of MATs. Figure 6 displays the Vehicle OBU node model developed in OPNET Modeller. It is worth noting that the new module is listed among node attributes as vho with two values (Enabled and Disabled).

\section{B. VhoMGT process model design}

The designed VhoMGT process model consists of two finite state machines (FSM) (see Fig. 7). The init state 


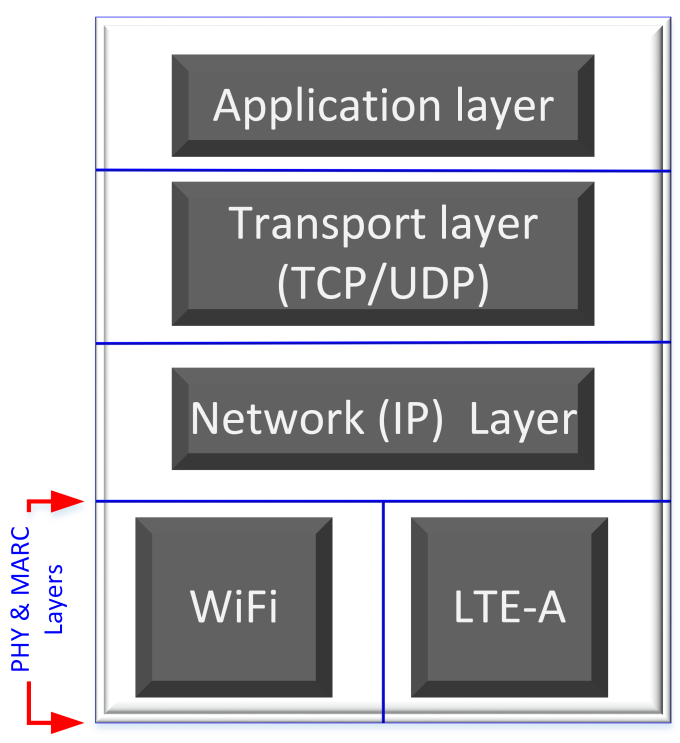

(a) OBU without new algorithm

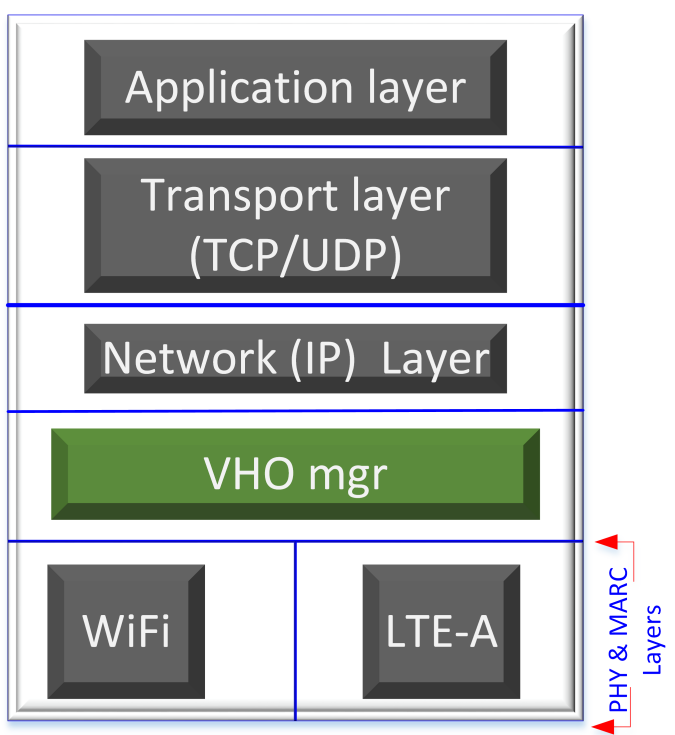

(b) OBU with new algorithm

Fig. 5. The TCP/IP Protocol Stack of On-Board Unit (OBU) Node

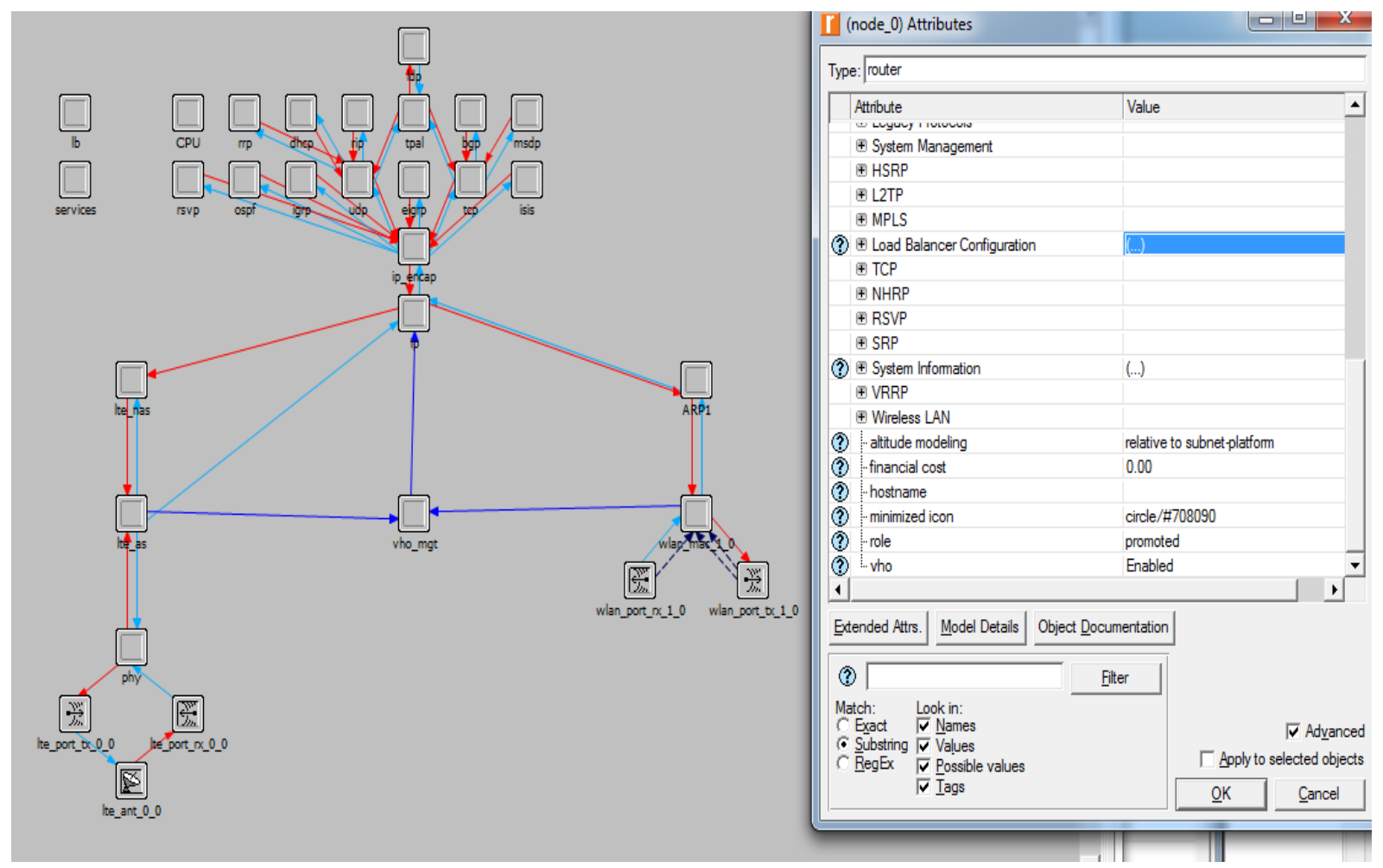

Fig. 6. Vehicle OBU node model developed in OPNET Modeler 


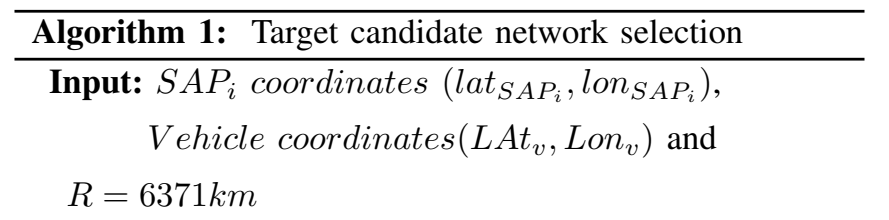

Output: Dwelling time $\left(t_{r}\right)$

while UE is connected to Macro Cell BS, do

Get current vehicle position, $p_{1}$

Get $l a t_{S A P_{i}}, \operatorname{lon}_{S A P_{i}}$, lat $_{v}, \operatorname{lon}_{v}$ and $R$

$\Delta_{\text {lat }_{1}}=$ lat $_{S A P_{i}}-$ lat $_{v_{p_{1}}}$

$\Delta_{l o n_{1}}=\operatorname{lon}_{S A P_{i}}-\operatorname{lon}_{v_{p_{1}}}$

$X=$

$\sin ^{2}\left(\frac{\Delta_{l a t_{1}}}{2}\right)+\cos \left(l_{a t_{v_{1}}}\right) * \cos \left(\right.$ lat $\left._{S A P_{i}}\right) * \sin ^{2}\left(\frac{\Delta_{l o n_{1}}}{2}\right)$

$d_{i 1}=2 R \sin (\sqrt{X}$

Set New Position $=P_{2}=\frac{d_{i 1}}{2}$

$\Delta_{l a t_{2}}=l_{\text {at }} t_{S A P_{i}}-$ lat $_{v_{p_{2}}}$

$\Delta_{l^{\prime o n_{2}}}=$ lon $_{S A P_{i}}-\operatorname{lon}_{v_{p_{2}}}$

$d_{i 2}=2 R \sin (\sqrt{X}$

$\gamma_{i}=d_{i 1}-d_{i 2} \backslash *$ Distance variation $*$

if $\gamma_{i} \leq 0$ then

$L$ delete $S A P_{i}$

else

foreach $\gamma_{i}>0$ do

$\delta d=P_{2}-P_{1}$

$\cos \alpha_{i}=\frac{d_{i 1}^{2}+\Delta d^{2}-d_{i 2}^{2}}{2 * d_{i 1} * \Delta d} \backslash *$ Cosine factor $*$

$t_{r_{i}}=\frac{C_{r} * \cos \alpha_{i}}{V_{\text {speed }}} \backslash *$ dwelling time*

return $t_{r_{i}}$

int[ ] SAPs $=$ new int[ ] $t_{r_{0}}, t_{r_{1}}, \ldots, t_{r_{n}} \backslash *$ list of

potential candidate SAPs*

Array.Sort (SAPs). $\backslash *$ descending order sorting*

if $\left(S A P s[0] . l i>=l i_{t h} \& \& S A P s[0] . R S S>=R S S_{t h}\right)$

then

HandoverEvent $=$ true $/ *$ Send handover command to

L macro-cell BS*/

else

HandoverEvent $=$ false $/ *$ Keep connection with macro cell $\mathrm{BS} * /$;

initializes all the necessary initializations. After initialization, the protocol will switch to the idle state and wait for any event from either LTE of Wifi MAC layers. The wait FSM executes code from the exit executive part of the state every time it receives events from LTE or WiFi interfaces.

\section{Simulation Setup}

Figure 8 shows the simulation scenario consisting of one LTE-A macro-cell eNB with greater coverage and two picocell HeNBs deployed at the edges of the macro-cell coverage area to provide better connectivity across a $2 \mathrm{Km} \times 2 \mathrm{Km}$ simulated terrain. The eNB and HeNBs are connected to the backbone traffic server through the Evolved Packet Core

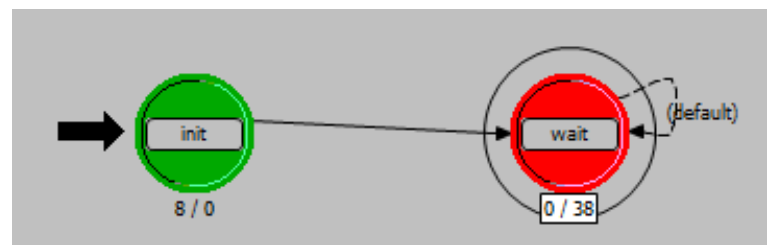

Fig. 7. The process model of the designed VHO management module.

TABLE I

SIMULATION PARAMETERS.

\begin{tabular}{|l|l|}
\hline Parameter & Values \\
\hline \hline Network area $(\mathrm{m} * \mathrm{~m})$ & $1000 * 1000$ \\
\hline $\begin{array}{l}\text { Transmit power of LTE } \\
\text { Macro/SAP }\end{array}$ & $0.5 \mathrm{~W} / 0.1 \mathrm{~W}$ \\
\hline LTE Macro/ SAP gain & $14 \mathrm{dBi} / 5 \mathrm{dBi}$ \\
\hline $\begin{array}{l}\text { WiFI SAP (IEEE 802.11p) } \\
\text { transmit power }\end{array}$ & $0.05 \mathrm{~W}$ \\
\hline Vehicle speed $(\mathrm{km} / \mathrm{h})$ & $20-120$ \\
\hline Path loss & $L$ \\
& $\left.10^{-3} \Delta h_{b}\right) \log _{10} R$ \\
$18 \log _{10} \Delta h_{b}+21 \log _{10} f+$ \\
$80) d B$
\end{tabular}

(EPC). Four Wi-Fi APs are also deployed randomly across the terrain sending information to the traffic server. A vehicle with the multi-mode OBU is simulated to move across this multi-tier HetNet environment performing handover with the most appropriate network selected as per the proposed method. Table 1 lists the simulation parameters used in this study.

The performance of the proposed network selection method is compared with (a) the conventional RSS-based method to select the most appropriate target network for handover, and (b) the Efficient ANDSF-assisted Wi-Fi Control for Mobile Data Offloading" method proposed in [19]. In the ANDSF-Assisted handover (HO) method in [10], the authors considered a heterogeneous environment consisting of Wi-Fi and cellular networks and have utilised a combination of the location coordinates of the neighbouring APs (provided 


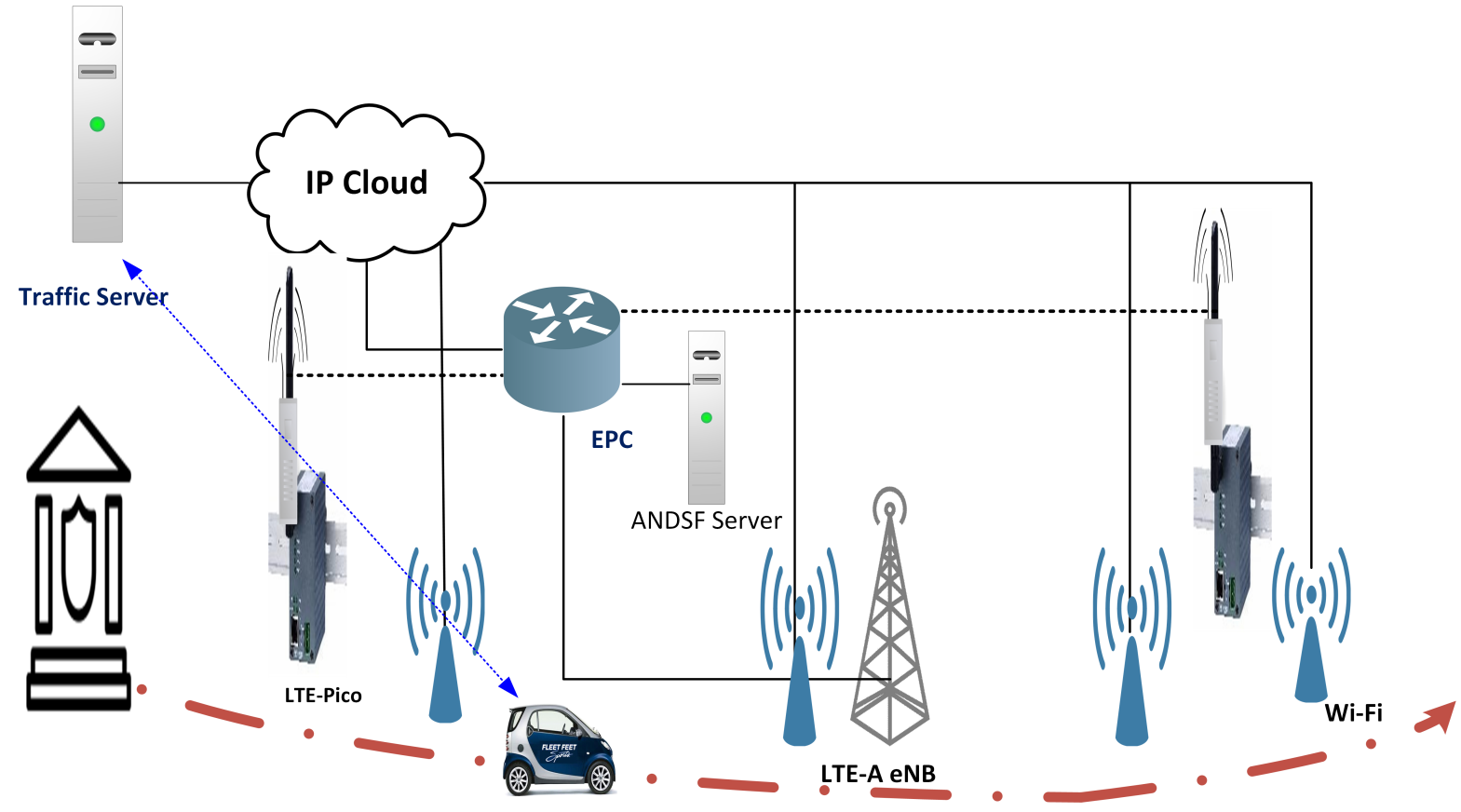

Fig. 8. The simulated network scenario

by the ANDSF server) and the measured RSS values to select the most appropriate network for handover. To evaluate and compare the performance of our proposed method, the following parameters are considered: number of handover performed, handover latency, handover failure rate (HOFR), throughput and end-to-end packet delay.

\section{Justification of Mobility Model}

In our OPNET simulation model we have considered Vector-based Trajectories as mobility model of vehicles because of its popularity and appropriateness in vehicular environment [22]. This vector-based mobility model has several advantages. First, it offers both the direction of movement and velocity for system performance study using Riverbed (OPNET) Modeler (simulation software). It should be noted that we have used OPNET because it is a credible simulation tool which has been tested by many network researchers and engineers worldwide. Second, the parameters such as direction of movement and velocity in the chosen vector-based mobility model can be changed at run time allowing us to experiment and study the system performance in a realistic vehicular scenario.

In this paper we focused on and spent a bit of time to develop a dual-mode vehicle On-Board Unit (OBU) containing both Long Term Evolution-Advanced (LTE-A) and WiFi interface cards for use in the simulation to study handover performance in multi-tier HetNet environment. Therefore, we did not study the impact of localization errors on system performance and we left it as our future work.

\section{E. Results and Discussions}

This sub-section discusses the simulation results to evaluate the performance analysis of the proposed network selec- tion method for handover based on the parameters mentioned above. In each case a comparison with the conventional RSSbased method and the ANDSF-Assisted handover method is presented for the vehicular movements along the projected trajectory at speeds ranging between $20 \mathrm{Km} / \mathrm{hr}$ to $120 \mathrm{Km} / \mathrm{hr}$. The simulation results are based on handover performance. Various metrics are investigated to prove the efficiency of the proposed handover method compared to the conventional mechanism. The simulation results report the steady state behavior of network and were obtained with a relative statistical error $1 \%$ at $99 \%$ confidence interval.

(i) Number Of Handovers Performed: Selection of an underlying network, which is not the most appropriate one for a handover activity can result in an increase in the number of unwanted handovers and lead to packet losses and even call drops. Generally, an increased number of handover activities affects the quality of the ongoing communication and hampers the overall handover performance. Figure 9 compares the results of the number of handovers performed by our proposed method with the conventional and the ANDSF-Assisted handover methods. As depicted in the results, the number of handovers performed by the vehicular OBU in our proposed method is less compared to the other two methods. While, depending on its speed of movement, the vehicle performed on an average nearly between 5 to 6 handovers in case of the conventional method and between 3 to 4 handovers in the ANDSF-Assisted HO method, it only performed between 2.5 to 3.5 handovers in our proposed method. Thus, our proposed method has managed to reduce the number of handovers by approximately $50 \%$ and $28 \%$, respectively, in compar- 


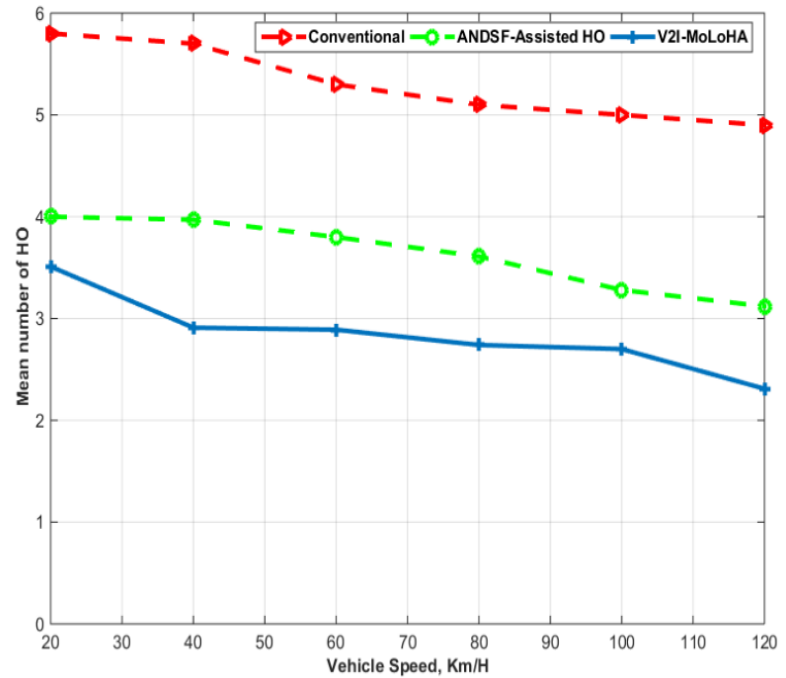

Fig. 9. Mean number of handovers versus vehicle speed performance comparison of conventional, ANDSF-Assisted, and the proposed methods

ison to the conventional and the ANDSF-Assisted HO methods. This is because to select the most appropriate target network for handover, in the proposed method the vehicle OBU scanned and considered only those SAPs that are located in its direction of movement and provide with a reasonable residence time with respect to the speed of the vehicle. On the contrary, in case of the other two methods, the vehicle OBU scanned and considered all advertised networks regardless of its direction of movement. Please note in this context that the number of performed handovers generally decreases with the increase in the vehicular speed of movement. This relates to different factors including Doppler shift effects and the limited cell coverage area of the small cells that does not allow the completion of handover activities for high speed vehicles. This leads to the increased handover failure rate exhibited in Figure 10.

(ii) Handover Failure Ratio (HOFR): The handover failure (HOF) occurs when a handover process is initiated but not carried out to the completion. The parameter HOFR is defined as the number of HOF to the total number of handover attempted (both successful and failure) [22]. The HOFR is measured in percentage as shown in (8).

$$
\operatorname{HOFR}(\%)=\frac{N_{H O F}}{N_{\text {SuccessHO }}+N_{H O F}}
$$

Higher handover failure rate deteriorates the expected QoS of ongoing communications, particularly for real time applications. The simulation results presented in the Figure 10 exhibited how much the proposed handover method reduces the percentage of HOFR. This is because the proposed network selection method considers the availability of adequate network resources at the target network prior to initiating the handover activities. This is well confirmed by the results depicted

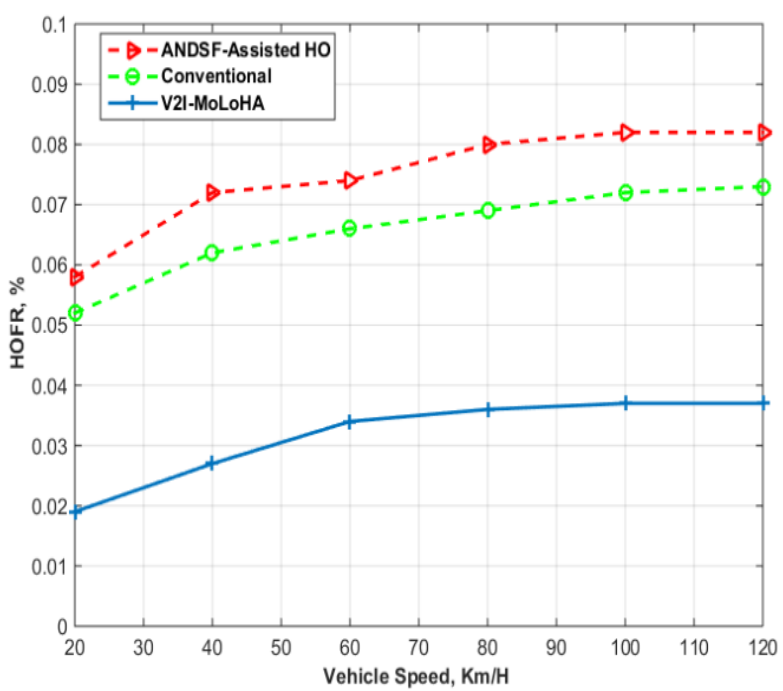

Fig. 10. Handover failure ratio (HOFR) versus vehicle speed performance comparison of conventional, ANDSF-Assisted, and the proposed methods

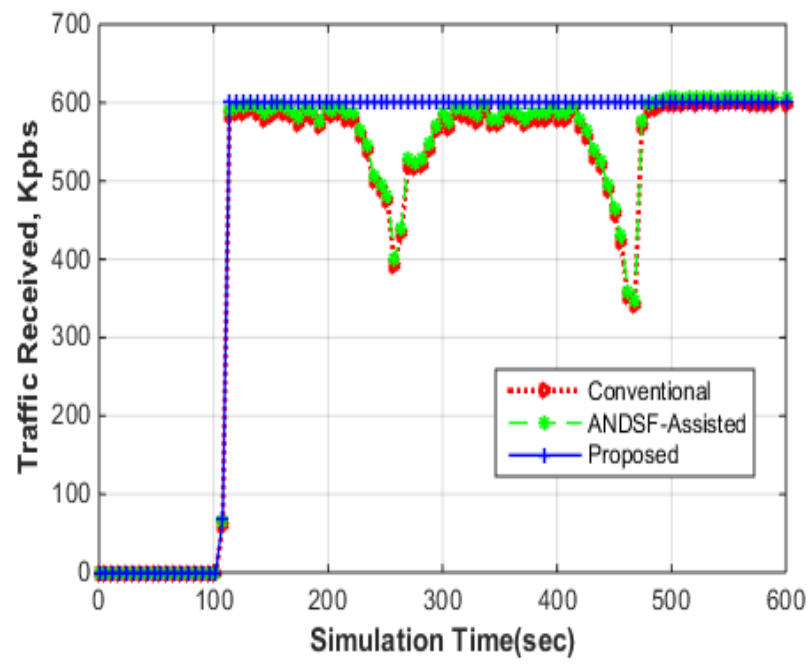

Fig. 11. Downlink traffic received by an On-Board Unit from an application server at vehicle speed of $60 \mathrm{~km} / \mathrm{h}$. Comparison of conventional, ANDSFAssisted, and the proposed handover methods

in the down-link traffic received by vehicle OBU from the traffic application server (See Figure 11). The fluctuation in the amount of traffic in case of conventional and ANDSF-Assisted scenarios is associated with increase of HOFR. On the contrary, the proposed method improves and stabilizes the download traffic received by the vehicle OBU from the traffic server due the optimised network selection decision that considers multiple selection parameters. HOFR in the proposed method is improved by approximately $50 \%$ in comparison to the conventional handover method and around $20 \%$ in comparison to the ANDSF-Assisted handover method.

(iii) Handover Latency: This parameter indicates the elapsed time between the initiation (from when a vehi- 


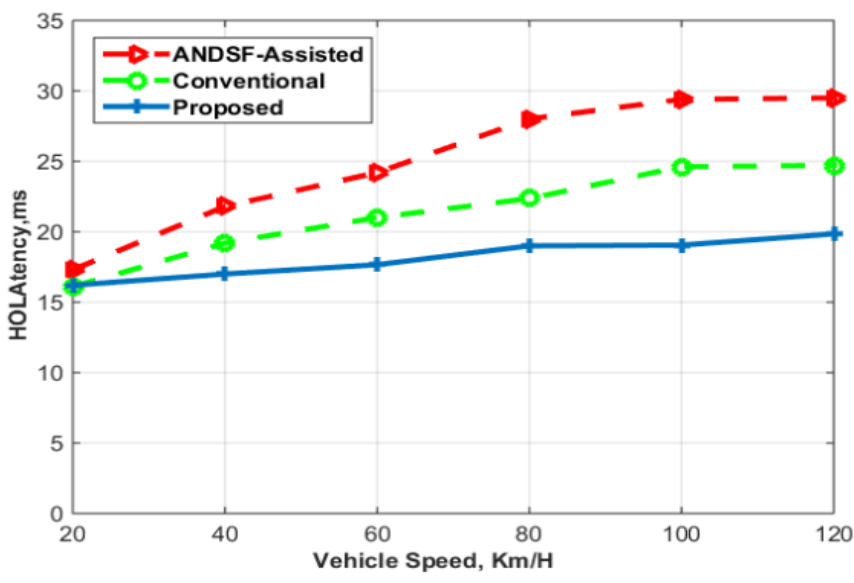

Fig. 12. Handover latency versus vehicle speed performance comparison of conventional, ANDSF-Assisted, and the proposed methods

cle feels the need for a handover) and the completion of a handover process Figure 12 compares the handover latency between the three considered methods. The reduction in the overall handover latency in our proposed method is owing to the reduced time that the vehicle OBU has spent in scanning only the potential target SAPs shortlisted based on the direction of movement, the residence time and the network load. As evident in Figure 12, our proposed method has managed to reduce the overall handover latency by approximately $40 \%$ and $20 \%$, respectively, in comparison to the conventional and the ANDSF Assisted HO methods. However, it is noted that the increase in the speed of movement implies the increased handover latency. This is due to the rise in radio link failures (RLF). These RLFs may occur due to the fact that the vehicle OBU crosses the boundaries of the serving cell before completing the handover activity to the target network. The increase in the handover latency is introduced by a RLF recovery process that involves selection of another best cell or re-selecting the previous eNB/AP.

(iv) OBU Throughput: Figure 13 compares mean throughput of conventional, ANDSF-Assisted, and the proposed method for vehicle speed ranging from 20 $\mathrm{Km} / \mathrm{h}$ to $120 \mathrm{Km} / \mathrm{h}$. As shown in Figure 13, the mean throughout proposed method is significantly better than both the conventional and ANDSF-Assisted handover. For example, the proposed method offers up to $50 \%$ and up to $20 \%$ higher throughput than the conventional and ANDSF-Assisted handover methods, respectively. The higher throughput is achieved due to less packet dropping owing to the reduced number of handovers offered by the proposed method. We observe that throughputs remain unaffected for both the proposed method and ANDSF-Assisted for vehicle speed; $20 \mathrm{Km} / \mathrm{h}$. However, throughput deteriorates with increasing vehicle speed. This throughput performance degradation is as a result of increased number of unsuc-

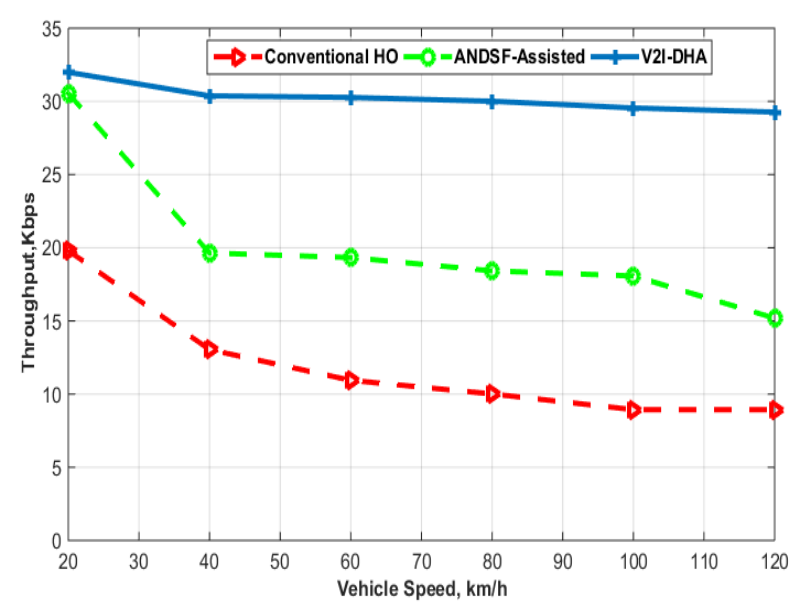

Fig. 13. Throughput versus vehicle speed performance for three network scenarios. Comparison of conventional, ANDSF-Assisted, and the proposed handover methods

cessful handovers and incomplete handover signalling messages between the vehicle OBU and the selected target networks caused by high vehicle speed. One can conclude that the network throughput is inversely impacted by vehicle speed when performing handovers to small cell networks.

(v) End-to-End Packet Delay: Figure 14 the end-to-end packet delay experienced by the vehicle OBU when running voice applications. End-to-end delay also called "mouth-to-ear" delay is measured from the ingress of the vehicle at the sender side to the egress of the vehicle at the receiver side [23]. A higher end-to-end packet delay affects the QoS performance of delay-sensitive user applications, like voice applications. End-to-end packet delay is a summation of various other delays, which include, network_delay, encoding_delay, decoding_delay, compression_delay, decompression_delay and dejitter_buffer_delay [23]. Our approach, however, is focused on reducing the network_delay, which is achieved by reducing the number of candidate SAPs to be scanned based on the proposed target network selection method. Moreover, the proposed method minimises the networ $k_{d}$ elay by starting network selection well in advance while approaching the candidate SAP. According to the results in Figure 14, our proposed target network selection method for handover is able to reduce the end-to-end packet delay by approximately $43 \%$ and $21 \%$, respectively, in the conventional and the ANDSFAssisted handover methods for a-voice application.

\section{CONCLUSION}

In this paper, we investigated the problem of selecting the most appropriate target network for handover in V2I communication over a multi-tier heterogeneous network is proposed. We proposed and derived new metrics, namely, the 


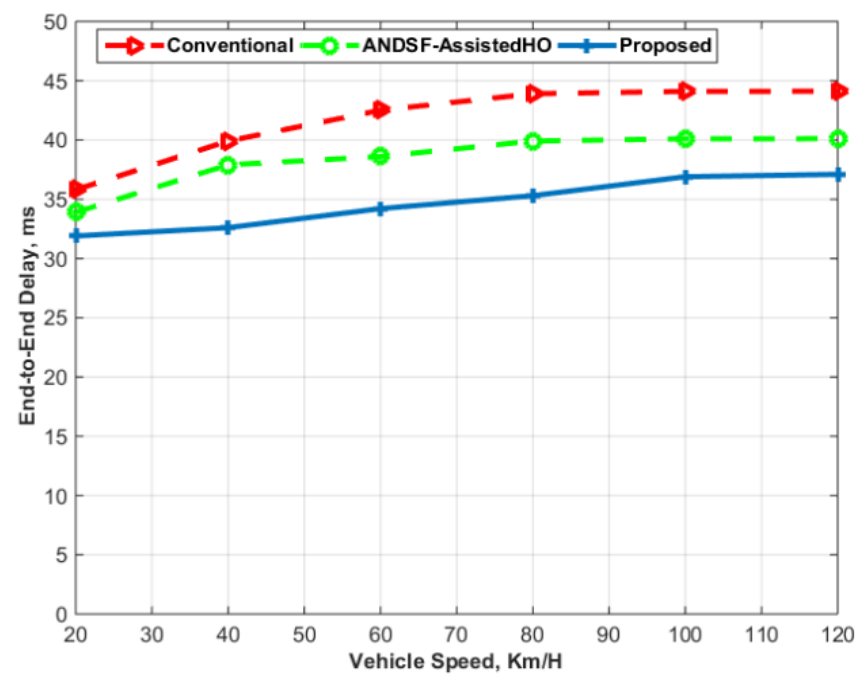

Fig. 14. End-to-end delay versus vehicle speed comparison of conventional, ANDSF-Assisted, and the proposed methods

relative direction index, proximity index, residence time, and network load index utilised for selecting the best candidate network. We developed and tested a dual-mode OBU (both LTE-A and Wi-Fi interfaces) in OPNET simulator. Vehicles equipped with these OBUs interfaces can select the most appropriate target network based on the above mentioned parameters and RSS values when moving across multitier heterogeneous urban networks. The performance of the proposed method is compared against the conventional and ANDSF-Assisted handover methods. Simulation results obtained have shown that the proposed approach can reduce the number of unnecessary handovers up to $50 \%$, mean handover latency and handover failure rate are also reduced up to $40 \%$. The proposed system offers up to $50 \%$ higher throughput and about $43 \%$ lower end-to-end delay than the conventional handover method. The application-aware network selection method is our future work.

\section{REFERENCES}

[1] Xiaohu Ge et al. "5G ultra-dense cellular networks". In: IEEE Wireless Communications 23.1 (2016), pp. 72-79.

[2] Ekram Hossain et al. "Evolution toward 5G multi-tier cellular wireless networks: An interference management perspective". In: IEEE Wireless Communications 21.3 (2014), pp. 118-127.

[3] Antonio Iera, Antonella Molinaro, and Salvatore Marano. "Handoff management with mobility estimation in hierarchical systems". In: IEEE Transactions on Vehicular Technology 51.5 (2002), pp. 915-934.

[4] Emmanuel Ndashimye et al. "Vehicle-to-infrastructure communication over multi-tier heterogeneous networks: a survey". In: Computer Networks (2016).
[5] Lina Xu et al. "Enterprise LTE and WiFi interworking system and a proposed network selection solution”. In: Proceedings of the 2016 Symposium on Architectures for Networking and Communications Systems. ACM. 2016, pp. 137-138.

[6] Tianqing Zhou et al. "Load-aware user association with quality of service support in heterogeneous cellular networks". In: IET Communications 9.4 (2015), pp. 494-500.

[7] ETSITS124312. "Universal mobile telecommunications system (UMTS), LTE; ac- cess network discovery and selection functions (ANDSF) management object (MO)". In: Release 12 3GPP TS 24.312 version 12.10.0 (Oct. 2015).

[8] Young Min Kwon et al. "Andsf-based congestion control procedure in heterogeneous networks". In: Information Networking (ICOIN), 2013 International Conference on. IEEE. 2013, pp. 547-550.

[9] Shun-Neng Yang et al. "Mobility management through access network discovery and selection function for load balancing and power saving in software-defined networking environment". In: EURASIP Journal on Wireless Communications and Networking 2016.1 (2016), p. 204.

[10] Dae Sun Kim et al. "Efficient ANDSF-assisted Wi$\mathrm{Fi}$ control for mobile data offloading". In: Wireless Communications and Mobile Computing Conference (IWCMC), 2013 9th International. IEEE. 2013, pp. 343-348.

[11] IEEE 802.11 Working Group et al. "IEEE Standard for Information Technology-Telecommunications and information exchange between systems-Local and metropolitan area networks-Specific requirementsPart 11: Wireless LAN Medium Access Control (MAC) and Physical Layer (PHY) specifications Amendment 9: Interworking with External Networks". In: IEEE Std 802.11 (2011).

[12] STEPHEN Rayment and J Bergstrom. "Achieving carrier-grade Wi-Fi in the 3GPP world". In: Ericsson Review (2012), pp. 2-7.

[13] Altice Labs. "Hotspot 2.0 and ANDSF for Smart Mobile User Connectivity". In: 2014. URL: http : / / www . alticelabs . com / content / WP Hotspot 2 - 0 - and - ANDSF - for - Smart Mobile-User-Connectivity.pdf.

[14] Huei-Wen Ferng and Ya-Yu Huang. "Handover scheme with enode-B pre-selection and parameter self-optimization for LTE-A heterogeneous networks". In: Machine Learning and Cybernetics (ICMLC), IEEE International Conference on. Vol. 2. 2016, pp. 594-599.

[15] Sanam Sadr and Raviraj S Adve. "Handoff rate and coverage analysis in multi-tier heterogeneous networks". In: IEEE Transactions on Wireless Communications 14.5 (2015), pp. 2626-2638.

[16] Shangguang Wang et al. "A vertical handoff method via self-selection decision tree for internet of ve- 
hicles". In: IEEE Systems Journal 10.3 (2016), pp. 1183-1192.

[17] Dionysis Xenakis et al. "Handover decision for small cells: Algorithms, lessons learned and simulation study". In: Computer Networks 100 (2016), pp. 64-74.

[18] Emmanuel Ndashimye, Nurul I Sarkar, and Sayan Kumar Ray. "A mobility-aware network selection method for vehicle-to-infrastructure communication over LTEA multi-tier networks". In: Information Networking (ICOIN), 2017 International Conference on. IEEE. 2017, pp. 315-320.

[19] Sokratis Barmpounakis et al. "Context-aware, userdriven, network-controlled RAT selection for 5G networks". In: Computer Networks 113 (2017), pp. 124147.

[20] Sayan Kumar Ray et al. "Self-tracking mobile station controls its fast handover in mobile WiMAX". In: Wireless communications and networking conference (WCNC), 2010 IEEE. IEEE. 2010, pp. 1-6.

[21] Riverbed-Technology. OPNET moduller. URL: http: / / www . riverbed . com / products / performance - management - control / opnet. html ? redirect $=$ opnet.

[22] Yaseein Soubhi Hussein et al. "A novel cell-selection optimization handover for long-term evolution (LTE) macrocellusing fuzzy TOPSIS". In: Computer Communications 73 (2016), pp. 22-33.

[23] Myasar R Tabany, Chris G Guy, and R Simon Sherratt. "A novel downlink semi-persistent packet scheduling scheme for VoLTE traffic over heterogeneous wireless networks". In: EURASIP Journal on Wireless Communications and Networking 2017.1 (2017), p. 62. 